1 Hacettepe Journal of Mathematics and Statistics

$\bigcap$ Volume 45 (3) (2016), 675-682

\title{
On the $P$-interiors of submodules of Artinian modules
}

\author{
H. Ansari-Toroghy ${ }^{* \dagger}$, F. Farshadifar ${ }^{\ddagger}$, and S. S. Pourmortazavi ${ }^{\S}$
}

\begin{abstract}
Let $R$ be a commutative ring and $M$ an Artinian $R$-module. In this paper, we study the dual notion of saturations (that is, $P$-interiors) of submodules of $M$ and obtain some related results.
\end{abstract}

Keywords: Second submodule, saturation, $P$-interior.

2000 AMS Classification: 13C13, 13C99, 13E10

Received: 15.12.2014 Accepted: 14.15.2015 Doi : 10.15672/HJMS.20164513104

\section{Introduction}

Throughout this paper, $R$ will denote a commutative ring with identity and " $\subset$ " will denote the strict inclusion. We write $N \leq M$ to indicate that $N$ is a submodule of an $R$-module $M$. Also $\operatorname{Spec}(R)$ and $\mathbb{Z}$ will denote the set of all prime ideals of $R$ and the ring of integers respectively.

Let $M$ be an $R$-module. A proper submodule $P$ of $M$ is said to be prime if for any $r \in R$ and $m \in M$ with $r m \in P$, we have $m \in P$ or $r \in\left(P:_{R} M\right)$. A non-zero submodule $S$ of $M$ is said to be second if for each $a \in R$, the endomorphism $S \stackrel{a}{\rightarrow} S$ is either surjective or zero (see [13]). A submodule $N$ of $M$ is said to be completely irreducible if $N=\bigcap_{i \in I} N_{i}$, where $\left\{N_{i}\right\}_{i \in I}$ is a family of submodules of $M$, implies that $N=N_{i}$ for some $i \in I$. It is easy to see that every submodule of $M$ is an intersection of completely irreducible submodules of $M$. Thus, the intersection of all completely irreducible submodule of $M$ is zero (see [6]).

*Department of pure Mathematics, Faculty of mathematical Sciences, University of Guilan, P. O. Box 41335-19141, Rasht, Iran.

Email : ansari@guilan.ac.ir

${ }^{\dagger}$ Corresponding Author.

${ }^{\ddagger}$ University of Farhangian, Tehran, Iran.

Email : f.farshadifar@gmail.com

${ }^{\S}$ Department of pure Mathematics, Faculty of mathematical Sciences, University of Guilan, P. O. Box 41335-19141, Rasht, Iran.

Email : mortazavi@phd.guilan.ac.ir 
The saturation of $N \leq M$ with respect to $P \in \operatorname{Spec}(R)$ is the contraction of $N_{P}$ in $M$ and designated by $S_{P}(N)$. It is well known that

$$
S_{P}(N)=\{e \in M: e s \in N \text { for some } s \in R-P\} .
$$

In [1], H. Ansari-Toroghy and F. Farshadifar, introduced the dual notions of saturations of submodules, that is, $P$-interiors of submodules and investigated some related results (see [1] and [3]). Let $N$ be a submodule of $M$. The $P$-interior of $N$ relative to $M$ is defined $[1,2.7]$ as the set

$$
\begin{gathered}
I_{P}^{M}(N)=\cap\{L \mid L \text { is a completely irreducible submodule of } M \text { and } \\
r N \subseteq L \text { for some } r \in R-P\} .
\end{gathered}
$$

There are considerable results about saturation of a module with respect to a prime ideal in literature (see, for example, [7], [8], and [9]). It is natural to ask that to what extent the dual of these results hold. The purpose of this paper is to answer this question and provide more information about the $P$-interiors of submodules in case that our module is an Artinian module.

\section{P-interiors of submodules and related properties}

Recall that an $R$-module $L$ is said to be cocyclic if $L$ is a submodule of $E(R / m)$ for some maximal ideal $m$ of $R$, where $E(R / m)$ is the injective envelope of $R / m$ (see [14]).

2.1. Lemma. Let $L$ be a completely irreducible submodule of an $R$-module $M$ and $a \in R$. Then $\left(L:_{M} a\right)$ is a completely irreducible submodule of $M$.

Proof. This follows from the fact that a submodule $L$ of $M$ is a completely irreducible submodule of $M$ if and only if $M / L$ is a cocyclic $R$-module by [6] and that $M /\left(L:_{M}\right.$ $a) \cong(a M+L) / L$.

We use the following basic fact without further comment.

2.2. Remark. Let $N$ and $K$ be two submodules of an $R$-module $M$. To prove $N \subseteq K$, it is enough to show that if $L$ is a completely irreducible submodule of $M$ such that $K \subseteq L$, then $N \subseteq L$.

2.3. Lemma. Let $P \in \operatorname{Spec}(R)$ and $N$ be a submodule of an $R$-module $M$. If $M / I_{P}^{M}(N)$ is a finitely cogenerated $R$-module, then there exists $r \in R-P$ such that $r N \subseteq I_{P}^{M}(N)$.

Proof. Since $M / I_{P}^{M}(N)$ is finitely cogenerated, there exists a finite number of completely irreducible submodules $L_{1}, L_{2}, \ldots, L_{n}$ of $M$ such that $I_{P}^{M}(N)=\cap_{i=1}^{n} L_{i}$ and $r_{i} N \subseteq L_{i}$ for some $r_{i} \in R-P$. Set $r=r_{1} \ldots r_{n}$. Then $r N \subseteq I_{P}^{M}(N)$.

2.4. Theorem. Let $P \in \operatorname{Spec}(R)$ and $N$ be a submodule of an $R$-module $M$. Then we have the following.

(a) If $M$ is an Artinian $R$-module, then $I_{P}^{M}\left(I_{P}^{M}(N)\right)=I_{P}^{M}(N)$.

(b) If $M$ is an Artinian $R$-module, then $\operatorname{Hom}_{R}\left(R_{P}, I_{P}^{M}(N)\right)=\operatorname{Hom}_{R}\left(R_{P}, N\right)$.

(c) $A n n_{R}(N) \subseteq S_{P}\left(A n n_{R}(N)\right) \subseteq A n n_{R}\left(I_{P}^{M}(N)\right)$.

(d) If $M$ is an Artinian $R$-module, then $A n n_{R}\left(I_{P}^{M}(N)\right)=S_{P}\left(A n n_{R}\left(I_{P}^{M}(N)\right)\right)$.

Proof. (a) Clearly, $I_{P}^{M}\left(I_{P}^{M}(N)\right) \subseteq I_{P}^{M}(N)$. To prove the opposite inclusion, let $L$ be a completely irreducible submodule of $M$ such that $I_{P}^{M}\left(I_{P}^{M}(N)\right) \subseteq L$. By Lemma 2.3, there exists $r \in R-P$ such that $r I_{P}^{M}(N) \subseteq I_{P}^{M}\left(I_{P}^{M}(N)\right)$. Therefore, $r I_{P}^{M}(N) \subseteq L$. Again by Lemma 2.3, there exists $s \in R-P$ such that $s N \subseteq I_{P}^{M}(N)$. Hence $r s N \subseteq L$. It follows that $I_{P}^{M}(N) \subseteq L$, as required. 
(b) By Lemma 2.3, there exists $r \in R-P$ such that $r N \subseteq I_{P}^{M}(N)$. Now $r N \subseteq$ $I_{P}^{M}(N) \subseteq N$ implies that

$$
\operatorname{Hom}_{R}\left(R_{P}, r N\right) \subseteq \operatorname{Hom}_{R}\left(R_{P}, I_{P}^{M}(N)\right) \subseteq \operatorname{Hom}_{R}\left(R_{P}, N\right) .
$$

As $r \in R-P$, one can see that $\operatorname{Hom}_{R}\left(R_{P}, r N\right)=\operatorname{Hom}_{R}\left(R_{P}, N\right)$. Therefore, ${ }^{* *}$ $\operatorname{Hom}_{R}\left(R_{P}, N\right)=\operatorname{Hom}_{R}\left(R_{P}, I_{P}^{M}(N)\right)$.

(c) Clearly, $A n n_{R}(N) \subseteq S_{P}\left(A n n_{R}(N)\right)$. Now let $r \in S_{P}\left(A n n_{R}(N)\right)$. Then there exists $s \in R-P$ such that $r s \in \operatorname{Ann}_{R}(N)$ and so $r s N=(\mathbf{0})$. Thus for each $i \in I$, $r s N \subseteq L_{i}$, where $\left\{L_{i}\right\}_{i \in I}$ is the collection of all completely irreducible submodules of $M$. Hence $s N \subseteq\left(L_{i}:_{M} r\right)$ for each $i \in I$. This implies that $I_{P}^{M}(N) \subseteq\left(L_{i}:_{M} r\right)$ for each $i \in I$ because $\left(L_{i}:_{M} r\right)$ is a completely irreducible submodule of $M$ by Lemma 2.1. Therefore, $r I_{P}^{M}(N) \subseteq \cap_{i \in I} L_{i}=(\mathbf{0})$. Thus $r \in \operatorname{Ann}_{R}\left(I_{P}^{M}(N)\right)$.

(d) Clearly, $A n n_{R}\left(I_{P}^{M}(N)\right) \subseteq S_{P}\left(A n n_{R}\left(I_{P}^{M}(N)\right)\right.$. Now let $r \in S_{P}\left(A n n_{R}\left(I_{P}^{M}(N)\right)\right.$. Then there exists $s \in R-P$ such that $r s \in A n n_{R}\left(I_{P}^{M}(N)\right)$ and so $r s I_{P}^{M}(N)=(\mathbf{0})$. As $M$ is an Artinian $R$-module, there exists $t \in R-P$ such that $t N \subseteq I_{P}^{M}(N)$ by Lemma 2.3. Therefore, $\operatorname{str} N=(\mathbf{0})$. This implies that for each $i \in I, \operatorname{st} N \subseteq\left(L_{i}:_{M} r\right)$, where $\left\{L_{i}\right\}_{i \in I}$ is the collection of all completely irreducible submodules of $M$. Hence $I_{P}^{M}(N) \subseteq\left(L_{i}:_{M} r\right)$. Therefore, $r I_{P}^{M}(N) \subseteq \cap_{i \in I} L_{i}=(\mathbf{0})$. Hence $r \in A n n_{R}\left(I_{P}^{M}(N)\right)$, as required.

2.5. Definition. We say that a submodule $N$ of an $R$-module $M$ is cotorsion-free with respect to (w.r.t.) $P$ if $I_{P}^{M}(N)=N$, where $P \in \operatorname{Spec}(R)$.

2.6. Lemma. Let $N$ ba a submodule of an $R$-module $M$ and $P \in \operatorname{Spec}(R)$. If $N$ is cotorsion-free w.r.t. $P$, then $N$ is cotorsion-free w.r.t. $Q$ for each $Q \in V(P)$.

Proof. Since $P \subseteq Q, I_{P}^{M}(N) \subseteq I_{Q}^{M}(N)$. Therefore, $N=I_{P}^{M}(N) \subseteq I_{Q}^{M}(N) \subseteq N$. Hence $N=I_{P}^{M}(N)=I_{Q}^{M}(N)$ for each $Q \in V(P)$.

A non-zero $R$-module $M$ is said to be secondary if for each $a \in R$, the endomorphism $M \stackrel{a}{\rightarrow} M$ is either surjective or nilpotent (see [10]). Clearly, every second module is a secondary module.

2.7. Example. $\quad(1)$ If $P \in \operatorname{Spec}(R)$, then every $P$-secondary submodule of an $R$ module $M$ is cotorsion-free w.r.t. $P$ by $[4,2.8]$.

(2) The $\mathbb{Z}$-module $\mathbb{Z}_{p} \infty$ is cotorsion-free w.r.t. (0).

2.8. Corollary. Let $P \in \operatorname{Spec}(R)$ and $N$ be a submodule of an $R$-module $M$. If $N$ is cotorsion-free w.r.t. $P$, then $A n n_{R}\left(I_{P}^{M}(N)\right)=S_{P}\left(A n n_{R}\left(I_{P}^{M}(N)\right)\right)$.

Proof. The results follows from part (c) of Theorem 2.4 because $N=I_{P}^{M}(N)$.

The cosupport of an $R$-module $M$ [12] is denoted by $\operatorname{Cosupp}(M)$ and it is defined by

$$
\begin{aligned}
& \operatorname{Cosupp}(M)=\left\{P \in \operatorname{Spec}(R) \mid P \supseteq \operatorname{Ann}_{R}(L)\right. \text { for some cocyclic } \\
&\text { homomorphic image } L \text { of } M\} .
\end{aligned}
$$

2.9. Theorem. Let $P \in \operatorname{Spec}(R)$ and $N$ be a submodule of an Artinian $R$-module $M$. Then we have the following.

(1) $A n n_{R_{P}}\left(\operatorname{Hom}_{R}\left(R_{P}, N\right)\right)=\left(A n n_{R}\left(I_{P}^{M}(N)\right)\right)_{P}$.

(2) The following statements are equivalent.

(a) $\operatorname{Hom}_{R}\left(R_{P}, N\right) \neq(\mathbf{0})$.

(b) $A n n_{R}\left(I_{P}^{M}(N)\right) \subseteq P$.

(c) $I_{P}^{M}(N) \neq(\mathbf{0})$.

(d) $P \in \operatorname{Cosupp}_{R}(N)$. 
Proof. (1) By Theorem 2.4 (b), $\operatorname{Hom}_{R}\left(R_{P}, I_{P}^{M}(N)\right)=\operatorname{Hom}_{R}\left(R_{P}, N\right)$. It is easy to see that

$$
\left(A n n_{R}\left(I_{P}^{M}(N)\right)\right)_{P} \subseteq A n n_{R_{P}}\left(\operatorname{Hom}_{R}\left(R_{P}, I_{P}^{M}(N)\right) .\right.
$$

To see the reverse inclusion, we note that $I_{P}^{M}\left(I_{P}^{M}(N)\right)=\phi\left(\operatorname{Hom}_{R}\left(R_{P}, I_{P}^{M}(N)\right)\right)$ by $[2$, 2.15], where $\phi: \operatorname{Hom}_{R}\left(R_{P}, I_{P}^{M}(N)\right) \rightarrow I_{P}^{M}(N)$ is the natural homomorphism defined by $\phi(f)=f\left(1_{R_{P}}\right)$ for any $f \in \operatorname{Hom}_{R}\left(R_{P}, I_{P}^{M}(N)\right)$. Now by Theorem $2.4(\mathrm{a}), I_{P}^{M}(N)=$ $\phi\left(\operatorname{Hom}_{R}\left(R_{P}, I_{P}^{M}(N)\right)\right)$. But always we have

$$
A n n_{R}\left(\operatorname{Hom}_{R}\left(R_{P}, I_{P}^{M}(N)\right)\right) \subseteq A n n_{R}\left(\phi\left(\operatorname{Hom}_{R}\left(R_{P}, I_{P}^{M}(N)\right)\right) .\right.
$$

Hence $\operatorname{Ann}_{R}\left(\operatorname{Hom}_{R}\left(R_{P}, I_{P}^{M}(N)\right)\right) \subseteq \operatorname{Ann} n_{R}\left(I_{P}^{M}(N)\right)$. Therefore,

$$
A n n_{R_{P}}\left(\operatorname{Hom}_{R}\left(R_{P}, I_{P}^{M}(N)\right)\right) \subseteq\left(A n n_{R}\left(I_{P}^{M}(N)\right)\right)_{P},
$$

as required.

$(2)(a) \Leftrightarrow(d)$. By [12, 2.3], $\operatorname{Cosupp}_{R}(N)=V\left(\operatorname{Ann}_{R}(N)\right)$ and by [11, p. 130], $\operatorname{Cos}_{R}(N)=V\left(\operatorname{Ann}_{R}(N)\right)$, where $\operatorname{Cos}_{R}(N)=\left\{P \in \operatorname{Spec}(R): \operatorname{Hom}_{R}\left(R_{P}, N\right) \neq(\mathbf{0})\right\}$. Hence we get the equivalence $(a)$ and $(d)$.

$(b) \Rightarrow(c)$. This is clear.

$(a) \Rightarrow(b) . H_{R} m_{R}\left(R_{P}, N\right) \neq(\mathbf{0}) \Leftrightarrow A n n_{R_{P}}\left(\operatorname{Hom}_{R}\left(R_{P}, N\right)\right) \neq R_{P}$. Thus by using part (1), we have

$$
\operatorname{Hom}_{R}\left(R_{P}, N\right) \neq(\mathbf{0}) \Leftrightarrow\left(\operatorname{Ann}_{R}\left(I_{P}^{M}(N)\right)\right)_{P} \neq R_{P} \Leftrightarrow A n n_{R}\left(I_{P}^{M}(N)\right) \subseteq P .
$$

$(c) \Rightarrow(a)$. If $\operatorname{Hom}_{R}\left(R_{P}, N\right)=(\mathbf{0})$, then $\operatorname{Hom}_{R}\left(R_{P}, I_{P}^{M}(N)\right)=(\mathbf{0})$. Thus by [2, 2.15],

$$
I_{P}^{M}(N)=I_{P}^{M}\left(I_{P}^{M}(N)\right)=\phi\left(\operatorname{Hom}_{R}\left(R_{P}, I_{P}^{M}(N)\right)=(\mathbf{0}),\right.
$$

where $\phi: \operatorname{Hom}_{R}\left(R_{P}, I_{P}^{M}(N)\right) \rightarrow I_{P}^{M}(N)$ is the natural homomorphism defined by $\phi(f)=$ $f\left(1_{R_{P}}\right)$ for any $f \in \operatorname{Hom}_{R}\left(R_{P}, I_{P}^{M}(N)\right)$. This contradiction completes the proof.

We need the following lemma.

2.10. Lemma. $[7,2.2]$ Let $I$ be an ideal of $R$ and $P \in \operatorname{Spec}(R)$. Then the following statements are equivalent.

(a) $S_{P}(I)$ is a $P$-primary ideal of $R$.

(b) $\sqrt{S_{P}(I)}=P$.

(c) $P$ is a minimal prime ideal of $I$.

2.11. Theorem. Let $P \in \operatorname{Spec}(R)$ and $N$ be a submodule of an Artinian $R$-module $M$. Then the following statements are equivalent.

(a) $I_{P}^{M}(N)$ is a $P$-secondary submodule of $M$.

(b) $A n n_{R}\left(I_{P}^{M}(N)\right)$ is a $P$-primary ideal of $R$.

(c) $\sqrt{A n n_{R}\left(I_{P}^{M}(N)\right)}=P$.

In particular, $I_{P}^{M}(N)$ is $P$-second if and only if $A n n_{R}\left(I_{P}^{M}(N)\right)=P$.

Proof. $(a) \Rightarrow(b)$. This is clear.

$(b) \Rightarrow(a)$. Since $A n n_{R}\left(I_{P}^{M}(N)\right)$ is a $P$-primary ideal of $R$ and $I_{P}^{M}\left(I_{P}^{M}(N)\right)=I_{P}^{M}(N)$ by Theorem $2.4(\mathrm{a}), I_{P}^{M}(N)$ is a $P$-secondary submodule of $M$ by [4, 2.2].

$(b) \Rightarrow(c)$. This is elementary.

$(c) \Rightarrow(b)$. Put $I=A n n_{R}\left(I_{P}^{M}(N)\right)$. Then by Theorem $2.4(\mathrm{~d}), S_{P}(I)=I$. Now, we have $\sqrt{I}=P=\sqrt{S_{P}(I)}$ by the hypothesis. It follows from Lemma 2.10 that $S_{P}(I)$ is a $P$-primary ideal of $R$. Hence $I=S_{P}(I)=A n n_{R}\left(I_{P}^{M}(N)\right)$ is a $P$-primary ideal of $R$, as required. 
2.12. Definition. Let $M$ be an $R$-module, $(\mathbf{0}) \neq N \leq M$ and $P \in \operatorname{Spec}(R)$. We say the pair $(N, P)$ satisfies property $(* *)$ if $S_{P}\left(A n n_{R}(N)\right)=A n n_{R}\left(I_{P}^{M}(N)\right) \neq R$. We say the module $M$ satisfies property $(* *)$ if for every $(\mathbf{0}) \neq N \leq M$ and $P \in V\left(A n n_{R}(N)\right)$ the pair $(N, P)$ satisfies property $(* *)$.

2.13. Remark. (a) For every $N \leq M$ and $P \in \operatorname{Spec}(R)$, if $A n n_{R}(N) \nsubseteq P$, then $I_{P}^{M}(N)=(\mathbf{0})$ because there exists $r \in R-P$ such that $r N=(\mathbf{0})$. Hence for each $i \in I, r N \subseteq L_{i}$, where $\left\{L_{i}\right\}_{i \in I}$ is the set of all completely irreducible submodules of $M$. Therefore, $I_{P}^{M}(N) \subseteq \cap_{i \in I} L_{i}=(\mathbf{0})$. However, the converse is not true in general. As a counter example, take the $\mathbb{Z}$-module $\mathbb{Z}$ as $M, N=\mathbb{Z}$, and $P=(0)$.

(b) Let $M$ be an $R$-module, $(\mathbf{0}) \neq N \leq M$ and $P \in \operatorname{Spec}(R)$. If a pair $(N, P)$ satisfies property $(* *)$, then by part (a), we have $A n n_{R}(N) \subseteq P$.

2.14. Example. (a) The $\mathbb{Z}$-module $\mathbb{Z}$ does not satisfy property $(* *)$ because $(\mathbb{Z}$, (0)) does not satisfy this property.

(b) Let $N$ be a non-zero submodule of an $R$-module $M$ and let $P$ be a prime ideal of $R$. If $N$ is cotorsion-free w.r.t. $P$, then $(N, P)$ satisfies property $(* *)$. This is because $I_{P}^{M}(N)=N \neq(\mathbf{0})$ implies that $A n n_{R}\left(I_{P}^{M}(N)\right)=A n n_{R}(N) \neq R$ and hence by Corollary 2.8, we have

$$
A n n_{R}(N)=S_{P}\left(A n n_{R}(N)\right)=A n n_{R}\left(I_{P}^{M}(N)\right) \neq R .
$$

Moreover, not only $(N, P)$, but also $(N, Q)$ for each $Q \in V(P)$ satisfies property (**) by Lemma 2.6. In particular, every $P$-secondary submodule $S$ of $M$ and each $Q \in V(P)=V\left(A n n_{R}(S)\right)$ satisfies property $(* *)$ by Example 2.7.

2.15. Theorem. Every non-zero Artinian $R$-module $M$ satisfies property $(* *)$.

Proof. Let $(\mathbf{0}) \neq N \leq M$ and $P \in V\left(A n n_{R}(N)\right)$. By Lemma 2.3, there exists $t \in$ $R-P$ such that $t N \subseteq I_{P}^{M}(N)$. Now let $r \in A n n_{R}\left(I_{P}^{M}(N)\right)$. Then $r t N=(\mathbf{0})$. Hence $r \in S_{P}\left(A n n_{R}(N)\right)$. Thus $R \neq \operatorname{Ann}_{R}\left(I_{P}^{M}(N)\right) \subseteq S_{P}\left(A n n_{R}(N)\right)$. The reverse inclusion follows from Theorem 2.4 (c).

2.16. Remark. Those modules $M$ which satisfy property (**) are not necessarily Artinian. For example, every vector space $W$ satisfies property $(* *)$ even it is of infinite dimensional. This is due to that every non-zero subspace $U$ of $W$ is (0)-second with $V\left(A n n_{R}(U)\right)=\{(0)\}$.

2.17. Corollary. Let $M$ be an Artinian $R$-module, $(\mathbf{0}) \neq N \leq M$ and $P \in \operatorname{Spec}(R)$.

(1) The following statements are equivalent.

(a) $I_{P}^{M}(N)$ is a $P$-secondary submodule of $M$.

(b) $\sqrt{S_{P}\left(A n n_{R}(N)\right)}=P$.

(c) $P$ is a minimal prime ideal of $A n n_{R}(N)$.

(2) $I_{P}^{M}(N)$ is a $P$-second submodule of $M$ if and only if $S_{P}\left(A n n_{R}(N)\right)=P$.

In particular, if $A n n_{R}(N)=P$, then $I_{P}^{M}(N)$ is a $P$-second submodule of $M$.

Proof. The proof is straightforward from Theorem 2.11, Lemma 2.10, and Theorem 2.4.

\section{Maximal second submodules}

A submodule $N$ of an $R$-module $M$ is said to be a maximal second submodule of a submodule $K$ of $M$, if $N \subseteq K$ and there does not exist a second submodule $L$ of $M$ such that $N \subset L \subset K($ see [1]).

3.1. Lemma. Let $R$ be an integral domain and let $M$ be an Artinian non-zero $R$-module. 
(a) If $I_{(0)}^{M}(M) \neq(\mathbf{0})$, then $I_{(0)}^{M}(M)$ is a maximal (0)-second submodule of $M$ and it contains every (0)-second submodule of $M$.

(b) $I_{(0)}^{M}(M)=M$ if and only if $M$ is a (0)-second submodule of $M$.

Proof. (a) This follows from [1, 2.9] and [3, 2.10].

(b) This follows from part (a) and [3, 2.10].

3.2. Theorem. Let $R$ be an integral domain of dimension $1, M$ be a non-zero Artinian $R$-module and $(0) \neq P \in V\left(A n n_{R}(M)\right)$. Then $I_{P}^{M}\left(\left(0::_{M} P\right)\right)$ is a maximal second submodule of $M$ if and only if $I_{P}^{M}\left(\left(0:_{M} P\right)\right) \nsubseteq I_{(0)}^{M}(M)$.

Proof. Since $(0) \subset P \subseteq A n n_{R}\left(\left(0::_{M} P\right)\right), \operatorname{dim} R=1$, and $R$ is a domain, it follows that if $A n n_{R}\left(\left(0:_{M} P\right)\right) \neq R$, then $A n n_{R}\left(\left(0:_{M} P\right)\right)=P$. Hence $I_{P}^{M}\left(\left(0:_{M} P\right)\right)$ is a second submodule of $M$ by $[1,2.8]$.

Suppose that $I_{P}^{M}\left(\left(0:_{M} P\right)\right)$ is a maximal second submodule of $M$. Then there are two cases:

(i) $I_{P}^{M}((0: M P))=M$ and

(ii) $I_{P}^{M}\left(\left(0:_{M} P\right)\right) \neq M$.

In case (i), $M$ is a $P$-second submodule for $P \neq(0)$. Consequently, $I_{(0)}^{M}(M) \neq M$ by Lemma 3.1 (b). Hence $I_{P}^{M}\left(\left(0:_{M} P\right)\right) \nsubseteq I_{(0)}^{M}(M)$.

In case (ii), $I_{P}^{M}\left(\left(0:_{M} P\right)\right)$ is a proper maximal second submodule of $M$. Hence $M$ is not a second submodule, in particular, it is not a (0)-second submodule so that $I_{(0)}^{M}(M) \neq M$ by Lemma 3.1 (b) again. Thus if $I_{(0)}^{M}(M) \neq(\mathbf{0})$, then $I_{(0)}^{M}(M)$ is a proper maximal (0)-second submodule of $M$ by Lemma 3.1 (a). Consequently, $I_{P}^{M}\left(\left(0:_{M} P\right)\right) \nsubseteq I_{(0)}^{M}(M)$ by the maximality of $I_{P}^{M}((0: M P))$ in $M$. On the other hand, if $I_{(0)}^{M}(M)=(\mathbf{0})$, then obviously, $I_{P}^{M}\left(\left(0 ;_{M} P\right)\right) \nsubseteq I_{(0)}^{M}(M)$.

Conversely, suppose that $I_{P}^{M}\left(\left(0 ;_{M} P\right)\right) \nsubseteq I_{(0)}^{M}(M)$. Then clearly $I_{(0)}^{M}(M) \neq M$. Thus by Lemma 3.1 (b), $M$ is not a (0)-second submodule. To see that $I_{P}^{M}\left(\left(0:_{M} P\right)\right)$ is a maximal second submodule of $M$, let $K$ be a second submodule of $M$ such that $I_{P}^{M}\left(\left(0:_{M}\right.\right.$ $P)) \subseteq K \subseteq M$. Then

$$
(0) \subseteq A n n_{R}(M) \subseteq A n n_{R}(K) \subseteq A n n_{R}\left(I_{P}^{M}((0: M P))\right)=P .
$$

Since $\operatorname{dim} R=1$, the prime ideal $A n n_{R}(K)=(0)$ or $P$. If $A n n_{R}(K)=(0)$, then $K$ is a (0)-second submodule. However, $K \neq M$ because $M$ is not a (0)-second submodule as we have seen above. Since every proper (0)-second submodule contained in $I_{(0)}^{M}(M)$, we have that $I_{P}^{M}\left(\left(0::_{M} P\right)\right) \subseteq K \subseteq I_{(0)}^{M}(M) \neq(\mathbf{0})$ which contradicts to $I_{P}^{M}\left(\left(0:_{M} P\right)\right) \nsubseteq I_{(0)}^{M}(M)$. Therefore, $A n n_{R}(K)=P$, i.e., $K$ is a $P$-second submodule. Thus $K=I_{P}^{M}(K) \subseteq$

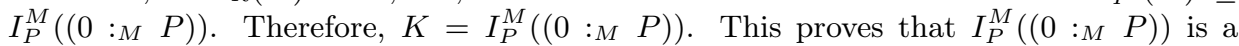
maximal second submodule of $M$.

3.3. Proposition. Let $Y$ be a set of prime ideals of $R$ which contains all the maximal ideals, $M$ be an Artinian $R$-module, and $N$ be a non-zero submodule of $M$. Then $N=\sum_{P \in Y} I_{P}^{M}(N)$.

Proof. Let $L$ be a completely irreducible submodule of $M$ such that $\sum_{P \in Y} I_{P}^{M}(N)$ $\subseteq L$ so that $I_{P}^{M}(N) \subseteq L$ for every $P \in Y$. Hence by Lemma 2.3 , we have $\left(L:_{R} N\right) \nsubseteq P$ for every $P \in Y$. This implies that $\left(L:_{R} N\right) \nsubseteq m$ for every maximal ideal $m \in Y$. This in turn implies that $\left(L:_{R} N\right)=R$ and hence $N \subseteq L$. Thus $N \subseteq \sum_{P \in Y} I_{P}^{M}(N)$. The reverse inclusion is clear. 
3.4. Corollary. Let $(R, m)$ be a local ring, $M$ an Artinian $R$-module, and $(\mathbf{0}) \neq N \leq M$. Then $N$ is cotorsion-free w.r.t. $m$.

Proof. Take $Y=\{m\}$ in Proposition 3.3. Then we have $I_{m}^{M}(N)=N$.

Let $N$ be a submodule of an $R$-module $M$. The (second) socle of $N$ is defined as the sum of all second submodules of $M$ contained in $N$ and it is denoted by $\operatorname{soc}(N)$ or $\sec (N)$ (see [1] and [5]). In case $N$ does not contain any second submodule, the socle of $N$ is defined to be $(\mathbf{0})$.

3.5. Proposition. Let $M$ be an Artinian $R$-module, $P \in \operatorname{Spec}(R)$, and $(\mathbf{0}) \neq N \leq M$. If $P$ is a minimal prime ideal of $A n n_{R}(N)$ and $I_{P}^{M}\left(\left(0:_{N} P\right)\right) \neq(\mathbf{0})$, then $I_{P}^{M}\left(\left(0:_{N} P\right)\right)$ is a maximal second submodule of $K \leq M$ with $I_{P}^{M}\left(\left(0:_{N} P\right)\right) \subseteq K \subseteq N$. In particular $I_{P}^{M}\left(\left(0:_{N} P\right)\right)$ is a maximal $P$-second submodule of $\sec (N)$.

Proof. Since $I_{P}^{M}\left(\left(0:_{N} P\right)\right) \neq(\mathbf{0}), I_{P}^{M}\left(\left(0:_{N} P\right)\right)$ is a maximal $P$-second submodule of $\left(0:{ }_{N} P\right)$ by $[1,2.9]$. Now suppose that $K$ is a submodule of $M$ such that $I_{P}^{M}\left(\left(0::_{N} P\right)\right) \subseteq$ $K \subseteq N$ and $S$ is a $Q$-second submodule of $M$ such that $I_{P}^{M}\left(\left(0:_{N} P\right)\right) \subseteq S \subseteq K \subseteq N$. Then as $P$ is a minimal prime ideal of $A n n_{R}(N)$, we have $Q=P$. Thus $S \subseteq\left(0:_{N} P\right)$. It follows that $S=I_{P}^{M}\left(\left(0:_{N} P\right)\right)$ as desired. The last assertion follows from the fact that $I_{P}^{M}\left(\left(0:_{N} P\right)\right) \subseteq \sec (N) \subseteq N$. So the proof is completed.

The following example shows that the condition $I_{P}^{M}\left(\left(0:_{N} P\right)\right) \neq(\mathbf{0})$ in the statement of Proposition 3.5 can not be dropped.

3.6. Example. Consider $M=N=\mathbb{Z}_{p^{\infty}}$ as $\mathbb{Z}$-module, where $p$ is a prime number. Let $q \neq p$ be an another prime number. Then clearly, $q \mathbb{Z}$ is a minimal prime ideal of $A n n_{\mathbb{Z}}(M)$ and $I_{(q)}^{M}\left(\left(0:_{N} q \mathbb{Z}\right)\right)=(\mathbf{0})$.

The next theorem gives an important information on the maximal second submodules of an Artinian $R$-modules.

3.7. Theorem. Let $N$ be a non-zero submodule of an Artinian $R$-module $M$. Then every maximal second submodule of $N$ must be of the form $I_{P}^{M}\left(\left(\begin{array}{lll}0:{ }_{N} & P\end{array}\right)\right)$ for some $P \in V\left(A n n_{R}(N)\right)$.

Proof. Let $S$ be a maximal $P$-second submodule of $N$. Then $S \subseteq N$ and $A n n_{R}(S)=P$ so that $S \subseteq\left(0::_{N} P\right)$. Therefore, $S=I_{P}^{M}(S) \subseteq I_{P}^{M}\left(\left(0:_{N} P\right)\right) \subseteq N$ by [3, 2.10]. Since $P \in V\left(A n n_{R}(N)\right), I_{P}^{M}\left(\left(0:_{N} P\right)\right)$ is a $P$-second submodule, as we have seen in the proof of Proposition 3.5. Thus $S=I_{P}^{M}\left(\left(0:_{N} P\right)\right)$.

3.8. Corollary. Let $M$ be an Artinian $R$-module and $(\mathbf{0}) \neq N \leq M$. Then $\sec (N)=$ $\sum_{P \in Y} I_{P}^{M}\left(\left(0:_{N} P\right)\right)$, where $Y$ is a finite subset of $V\left(A n n_{R}(N)\right)$.

Proof. By $[1,2.6,2.2]$, there exists $n \in \mathbb{Z}$ such that $\sec (N)=\sum_{i=1}^{n} S_{i}$, where for $1 \leq i \leq n, S_{i}$ is a maximal second submodule of $N$. Now the proof follows from Theorem 3.7. We remark that this corollary is also a direct consequence of [3, Proposition 2.7 (a)].

3.9. Corollary. Let $N$ be a non-zero submodule of an Artinian $R$-module $M$. If $I_{P}^{M}((0: N$ $P)) \neq(\mathbf{0})$ and $N$ is a $P$-secondary submodule of an $R$-module $M$ for some $P \in \operatorname{Spec}(R)$, then we have the following.

(a) $I_{P}^{M}((0: N P))$ is a maximal $P$-second submodule of $\sec (N)$.

(b) If $P$ is a maximal ideal of $R$, then $\sec (N)=I_{P}^{M}\left(\left(0:_{N} P\right)\right)$ so that $\sec (N)$ is a $P$-second submodule of $M$. 
Proof. (a) This follows from Proposition 3.5 because $P$ is a minimal prime ideal of $A n n_{R}(N)$.

(b) By Corollary 3.8, $\sec (N)=\sum_{Q \in V\left(A n n_{R}(N)\right)} I_{Q}^{M}\left(\left(0:_{N} Q\right)\right)$. Since $P$ is maximal and $\sqrt{A n n_{R}(N)}=P, V\left(A n n_{R}(N)\right)=\{P\}$. Thus $\sec (N)=I_{P}^{M}\left(\left(0:_{N} P\right)\right)$ as required.

3.10. Corollary. Let $I$ be an ideal of $R$ and $M$ be an Artinian $R$-module such that $\left(0:_{M} I\right) \neq(\mathbf{0})$. Then $\sec \left(\left(0:_{M} I\right)\right)=\sum_{P \in V\left(A n n_{R}\left(\left(0:_{M} I\right)\right)\right)} I_{P}^{M}\left(\left(0:_{M} P\right)\right)$.

Proof. Set $N=\left(0:_{M} I\right)$. Then this follows from Corollary 3.8 since, $\left(0:_{\left(0::_{M} I\right)} P\right)=$ $\left(0:_{M} P\right)$ for every $P \in V\left(\operatorname{Ann}_{R}\left(\left(0:_{M} I\right)\right)\right)$.

3.11. Example. For any prime integer $p$, let $M=(\mathbb{Z} / p \mathbb{Z}) \times \mathbb{Z}_{p} \infty$. Then $M$ is an Artinian faithful $\mathbb{Z}$-module and $V\left(A n n_{\mathbb{Z}}(M)\right)=V((0))=\operatorname{Spec}(\mathbb{Z})$. Hence $\sec (M)=$ $\sum_{(q) \in V((0))} I_{(q)}^{M}\left(\left(0:_{M} q \mathbb{Z}\right)\right)$ by Corollary 3.10. Since $I_{(q)}^{M}\left(\left(0:_{M} q \mathbb{Z}\right)\right)=I_{(q)}^{M}(\mathbf{0})=(\mathbf{0})$ for each prime number $p \neq q$,

$$
\begin{aligned}
\sec (M) & =I_{(0)}^{M}(M)+I_{(p)}^{M}\left(\left(0:_{M} p \mathbb{Z}\right)\right) \\
& =\left((0) \times \mathbb{Z}_{p} \infty\right)+((\mathbb{Z} / p \mathbb{Z}) \times<1 / p+\mathbb{Z}>) \\
& =M .
\end{aligned}
$$

Acknowledgments. We would like to thank the referees for the careful reading our manuscript and valuable comments.

\section{References}

[1] H. Ansari-Toroghy and F. Farshadifar, On the dual notion of prime submodules, Algebra Colloq. 19 (Spec 1)(2012), 1109-1116.

[2] H. Ansari-Toroghy and F. Farshadifar, The Zariski topology on the second spectrum of a module, Algebra Colloq. 21 (4) (2014), 671-688.

[3] H. Ansari-Toroghy and F. Farshadifar, On the dual notion of prime submodules (II), Mediterr. J. Math., 9 (2) (2012), 327-336.

[4] H. Ansari-Toroghy, F. Farshadifar, S.S. Pourmortazavi, and F. Khaliphe On secondary modules, Int. J. Algebra, 6 (16) (2012), 769-774.

[5] S. Ceken, M. Alkan, P.F. Smith, The dual notion of the prime radical of a module, J. Algebra. 392 (2013), 265-275.

[6] L. Fuchs, W. Heinzer, and B. Olberding, Commutative ideal theory without finiteness conditions: Irreducibility in the quotient filed, in: Abelian Groups, Rings, Modules, and Homological Algebra, Lect. Notes Pure Appl. Math. 249 (2006), 121-145.

[7] C.P. Lu, Saturations of submodules, Comm. Algebra 31 (6) (2003), 2655-2673.

[8] R.L. McCasland and P.F. Smith, Prime submodules of Noetherian modules, Rocky Mountain J. Math 23 (3) (1993), 1041-1062.

[9] R.L. McCasland and P.F. Smith, Generalised associated primes and radicals of submodules, Int. Electron. J. Algebra 4 (2008), 159-176.

[10] I.G. Macdonald, Secondary representation of modules over a commutative ring, Sympos. Math. XI (1973), 23-43.

[11] L. Melkersson and P. Schenzel, The co-localization of an Artinian module, Proc. Edinburgh Math. 38 (2) (1995), 121-131.

[12] S. Yassemi, Coassociated primes, Comm. Algebra. 23 (1995), 1473-1498.

[13] S. Yassemi, The dual notion of prime submodules, Arch. Math. (Brno) 37 (2001), 273-278.

[14] S. Yassemi, The dual notion of the cyclic modules, Kobe. J. Math. 15 (1998), 41-46. 